\title{
The Model of Degradable Polymer PLGA Deformed by Hot Embossing Method
}

\author{
Yang $\mathrm{Gao}^{1, \mathrm{a}}$, Hongyu Ge $\mathrm{G}^{1, \mathrm{~b}}$, Yaguang Huo ${ }^{2, \mathrm{c}}$ \\ ${ }^{1}$ School of Mechanical Engineering, Xi'an University of Science and Technology, Xi'an,710054, \\ China; \\ ${ }^{2}$ Modern Engineering Training Center, Chang'an University, Xi'an, 710064, China. \\ agaoyang_mec@163.com, b9203631@qq.com, chuoyg01@163.com
}

Keywords: Degradable polymer, hot embossing, simulation model, controlled drug delivery.

\begin{abstract}
A novel drug delivery microsystem is proposed in this paper, which consists of the microstructure for drug loading and the bonding membrane with microholes for release rate controlling. The forming process of microstructure by hot embossing method was researched. For the microstructure was fabricated by degradable polymer which possess the viscoelastic property, the numerical material model was established based on the generalized Maxwell model with 5 units, and the relaxation parameters in this model were confirmed by the stress relaxation experiment. The simulation results show that the model is reasonable and give the base for the optimum parameters research by hot embossing.
\end{abstract}

\section{Introduction}

Biomaterials have played an enormous role in the success of medical devices, especially in the development of controlled drug delivery systems (CDDS). Kinds of CDDS with different structures and application purposes have been developed, such as microcapsules used for insulin releasing [1], micro-spheres as a sustained release system for hormone [2], hydrogel composites as cell carriers [3], micro-spheres modulating the delivery of neurotrophic factors [4].

The stable release rate in the whole drug delivery process is very important. It is therefore our research team proposed a novel structure of degradable depots-composed CDDS, which fabricated by Polylactic acid-polyglycolic acid (PLGA). This CDDS has micro- $\square$ depot arrays for drug storage and holes at the micron-scale in its bonding membrane, which can get the linear cumulative release rate [5]. The maximum dimension of this CDDS in diameter is $10 \mathrm{~mm}$ and the drug-depot depth is 400-500 $\mu \mathrm{m}$. The schematic diagram of this novel CDDS is displayed in Fig. 1. The hot embossing method was chosen to produce the micro matrix which used for drug loading.

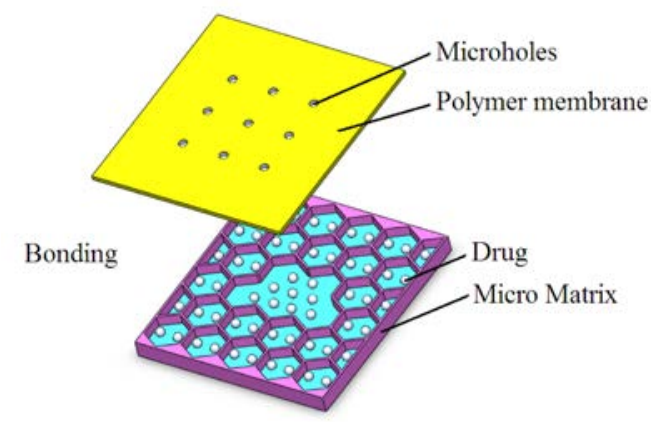

Fig. 1 Schematic of the biodegradable CDDSM

The formation of the microstructure is important because it is not only taking shape the drug loading cavities but also the part for bonding which makes the integrality of this CDDS. Bad shape of microstructure will cause the leaking of drugs, so the release rate will lose the control. Good quality pattern transfer depends on the tool characteristics, accurate temperature and pressure control during the embossing process. 
Since the polymer usually has macromolecular chains, so it is possesses stress relaxation and viscoelasticity in hot embossing process. The polymer of our research is PLGA, which glass transition temperature is $45-50{ }^{\circ} \mathrm{C}$, and the deformation properties is time-related because the specific entropy elasticity of polymer chains. But the research on the viscoelasticity of PLGA and the simulation of deformed process are less. The aim of this paper is to research the relaxation property of PLGA and establish the reasonable deformation model by the finite element method (FEM) to simulate the process of PLGA hot embossing.

\section{Viscoelastic Model of PLGA}

For the entropy elasticity of polymer macromolecular chains, the mechanical behavior of polymers above the glass transient temperature is not only temperature-dependent, but also time-dependent, presenting the viscoelastic property. In this situation, the temperature-dependent linear elastic model [6] and a nonlinear elastic model like the Mooney- Revilin model [7] are not suited. So, the generalized Maxwell model was used to describe the molding behavior of viscoelastic materials in hot embossing, in which springs and damps are simulated the linear elastic response and the linear viscous response respectively. Because the macromolecular chains of PLGA is entangled each other, so the ultimate relaxation modulus is not zero as the same as the linear polymer. In this situation, an extra spring, $G_{\infty}$, must be paralleled on the generalized Maxwell model. The schematic of PLGA deformation model is shown in Fig.2.

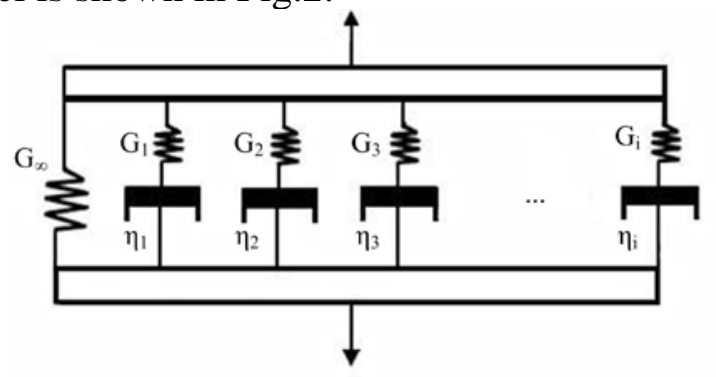

Fig.2 The schematic of PLGA deformation model

According to the Boltzmann superposition principle, the viscoelastic deformation of PLGA is commonly described by the following equation:

$$
\sigma(t)=\varepsilon_{0} G(t)+\int_{0}^{t} G\left(t-t^{\prime}\right) \dot{\varepsilon}\left(t^{\prime}\right) d t^{\prime}
$$

Where $\sigma(t)$ and $\dot{\varepsilon}(t)$ represent the time-dependent stress and the strain rate, respectively. $\varepsilon_{0}$ is the initial value of strain, $t$ and $t$ ' represent the current and past time.

The time-dependent relaxation modulus, $G(t)$, can be captured by a mechanical model with a sufficient number of elastic and viscous elements and expressed as exponential series:

$$
\begin{aligned}
& G(t)=G_{\infty}+\sum_{i=1}^{n} G_{i} \exp \left(-\frac{t}{\tau_{i}}\right) \\
& G_{0}=G(t=0)=G_{\infty}+\sum_{i=1}^{n} G_{i}
\end{aligned}
$$

Where $n$ is the number of damp-spring units, $G_{\infty}$ is the equilibrium value of $G(t)$ after the time goes to infinity, $\tau_{i}$ is the relaxation time. Until now, there are no research data about PLGA relaxation modulus, so the Stress relaxation experiments were carried out to confirm the values of Maxwell model. The PLGA material (monomer ratio PLA/PGA is 75/25) used in the stress relaxation experiments is purchased from LACTEL ${ }^{\circledR}$ USA. Based on the experimental results, the parameters of generalized Maxwell model with 5 units are listed in the Tab. 1. The elastic modulates of PLGA at different temperature are shown in Tab.2. 
Tab. 1 The parameters of generalized Maxwell model

\begin{tabular}{cccc}
\hline Parameters & Fitting value & Parameters & Fitting value \\
\hline$G_{\infty}$ & 0.00017 & -- & -- \\
$G_{1}$ & 0.16845 & $\tau_{1}$ & 1.21 \\
$G_{2}$ & 0.3181 & $\tau_{2}$ & 19.73 \\
$G_{3}$ & 0.31483 & $\tau_{3}$ & 113.27 \\
$G_{4}$ & 0.17621 & $\tau_{4}$ & 396.38 \\
$G_{5}$ & 0.05149 & $\tau_{5}$ & 1565.40 \\
\hline
\end{tabular}

Tab.2 The elastic modulus of PLGA at different temperatures

\begin{tabular}{ccccc}
\hline Temperature $/{ }^{\circ} \mathrm{C}$ & 65 & 70 & 75 & 80 \\
\hline Modulus/MPa & 2.32 & 1.99 & 1.40 & 0.97 \\
\hline
\end{tabular}

The finite element system ANSYS was used to model and simulate the formation behavior of PLGA substrate to microstructure in hot embossing process. The geometry model was contributed firstly, as shown in Fig.3. For the symmetry shape of micro structure, half of the groove template was taken as the analysis object. The deepness of template, $H$, is $540 \mu m$; the half of width, $L / 2$, is $60 \mu m$ and the half of spacing, $S / 2$, is $800 \mu m$.

In the analysis, supposed that there is no relative slip in the contact surface of the template and PLGA substrate. Boundary conditions at the left and the right boundary are symmetrical, the bottom of PLGA membrane was limited, and the constant pressure was applied on the template surface perpendicularly. The temperature distribution in this model is evenly. The material of template is PDMS, which elastic modulus is $3 \mathrm{MPa}$ and the Poisson's ratio is 0.499 .

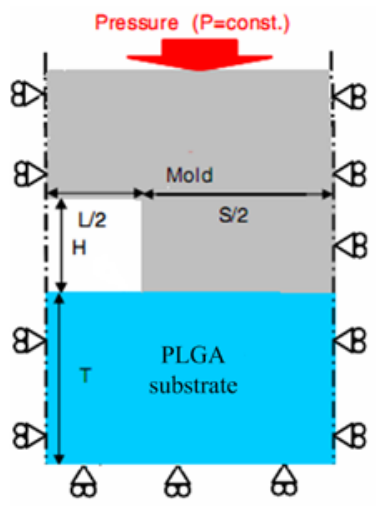

Fig. 3 The geometry model of microstructure formation

\section{The simulation of PLGA deformation}

The PLGA substrate filling the template groove under the pressure and the deformation degree is related with the action time of pressure. So, the influence of time-pressure on the microstructure formation was studied firstly. Fig.4 shows the same template groove was filled by PLGA at 80 ond under the acting pressure $20 \mathrm{~N}$ and $30 \mathrm{~N}$ respectively, the action time of pressure is $20 \mathrm{~s}$, 50 s and 70 s.

As shown in Fig.4, there is a tendency that the higher forming pressure and longer action time make better quality of the microstructure. For every forming pressure, the height of the filled polymer in template groove all increased with the elapsing of action time, but the filling speed is direct proportional with the pressure. 


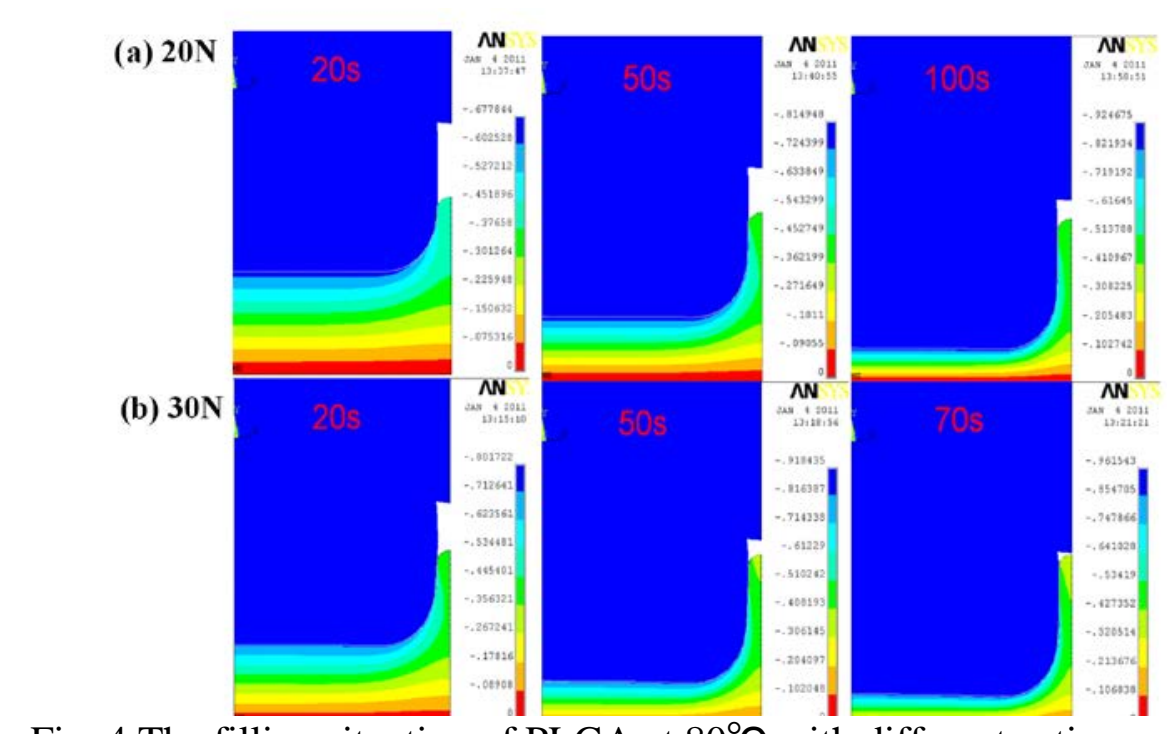

Fig. 4 The filling situation of PLGA at $80^{\circ} \mathrm{C}$ with different acting pressure

\section{Conclusions}

In this paper, the model was established to simulate the deformation of PLGA by hot embossing method, the property of stress relaxation was researched, and the relaxation modulus of 75/25 PLGA with the increasing temperatures was tested by experiment. The hot embossing process was simulated using FEM based on the generalized Maxwell mold with 5 units. The simulation results show that the model is reasonable, and the above researches give the bases for the parameters optimization of PLGA microstructure formation by hot embossing.

\section{Acknowledgments}

The authors gratefully acknowledge the support of China Postdoctoral Science Foundation (2015M572579), Scientific Research Program Funded by Shaanxi Provincial Education Department (Program No. 2013JK1031), Engagement Program (201224, 201227) and Doctoral Research and Start-up Program (2012QDJ026) Funded by Xi'an University of Science and Technology.

\section{References:}

[1] Yamaguchi Y, Takenaga M, Kitagawa A, et al. Insulin-loaded biodegradable PLGA microcapsules initial burst release controlled by hydrophilic additives. Journal of controlled release. 81 (2002) 235-249.

[2] Du L, Cheng JP, Chi Q, et al. Biodegradable PLGA microspheres as a sustained release system for a new luteinizing hormone-releasing hormone (LHRH) antagonist. Chemical and Pharmaceutical Bulletin. 54 (2006) 1259-1265.

[3] Wang F, Li Z, Khan M, et al. Injectable, rapid gelling and highly flexible hydrogel composites as growth factor and cell carriers. Acta Biomaterialia. 6 (2010) 1978-1991.

[4] Bertram JP, Rauch MF, Chang K, et al. Using polymer chemistry to modulate the delivery of neurotrophic factors from degradable microspheres: Delivery of BDNF. Pharmaceutical Research. 27 (2010) 82-91.

[5] Gao Y, Chen T, Wang X. Numerical modeling of a novel degradable drug delivery system with microholes. Microsystem Technologies. 17 (2011) 387-394.

[6] Fu G, Loh NH, Tor SB, et al. Analysis of demolding in micro metal injection molding. Microsystem Technologies. 12 (2006) 554-564.

[7] Hirai Y, Fujiwara M, Okuno T, et al. Study of the resist deformation in nanoimprint lithography. Journal of Vacuum Science and Technology B: Microelectronics and Nanometer Structures. 19 (2001) 2811-2815. 